\title{
Désirer, acheter, consommer. Approche lacanienne
} Desire, purchasing, consumption: A Lacanian approach

\author{
David Bernard*1 \\ Quentin Dumoulin*2
}

\begin{abstract}
Il s'agira ici de questionner la fonction que pour l'être parlant, peuvent occuper les pratiques d'achat et de consommation, encore peu étudiées en tant que telles dans le champ de la psychanalyse et de la psychopathologie. A partir de l'enseignement de Jacques Lacan, nous tâcherons de préciser la logique de ces pratiques, en isolant en quoi elles peuvent constituer pour un sujet une fausse réponse quant à l'embarras de son désir. De là, nous questionnerons aussi comment ces pratiques s'actualisent dans l'espace numérique du monde contemporain, et comment ce faisant elles participent au malaise actuel dans la civilisation.
\end{abstract}

Mots clés: Acheter, consommer, désirer, lien social 


\section{ARTIGOS}

\section{Introduction}

Nous souhaiterions questionner ici, à partir de l'enseignement du psychanalyste Jacques Lacan, le rapport de l'être parlant aux pratiques d'achat et de consommation, ainsi qu'à leur actualisation dans l'espace du numérique. Une telle étude permet en effet de mieux situer la problématique du désir au regard de ce que Freud (1929/1971) nomma le «malaise dans la civilisation», et de réfléchir à son devenir dans le monde contemporain. Lacan aura proposé dans son enseignement de nombreux commentaires à ce sujet. Il put notamment souligner la part prise par le discours capitaliste dans ce malaise, et souligner comment celui-ci est venu accentuer l'embarras voire l'égarement du sujet quant à son désir. La raison en tiendrait selon lui à ce qu'il nomme, dans une référence à Karl Marx, «l'exploitation du désir», à quoi procède ce discours. Ainsi, énonce-t-il en 1973 dans une conférence à Milan: «L'exploitation du désir, c'est la grande invention du discours capitaliste, parce qu'il faut quand-même l'appeler par son nom. Ça je dois dire, c'est un truc vachement réussi. Qu'on soit arrivé à industrialiser le désir, enfin... on ne pouvait rien faire de mieux pour que les gens se tiennent un peu tranquilles, hein? Et d'ailleurs, on en a obtenu le résultat. C'est beaucoup plus fort qu'on ne le croit» (Lacan, 1978). Il y a donc pour Lacan au fondement du malaise dans la civilisation la réussite du discours capitaliste, dont il propose ici une définition précise: ce discours est parvenu à exploiter et à industrialiser le désir.

Le capitalisme ne devrait donc pas son succès et son pouvoir à ce qu'il propose comme nouveaux objets, mais à sa façon d'exploiter la structure du désir, présente depuis toujours. Cette exploitation particulière le caractérise et le différencie des autres discours définis par Lacan (1969-1970/1991) comme régissant le lien social. Enfin son offre et son discours sont désormais diffusés mondialement par les outils numériques et l'on pourrait dire que la genèse de ces derniers ne peut se départir de l'histoire de leur commercialisation (Breton, 1990). L'évolution de ces «nouveaux objets», leurs sophistications techniques et esthétiques, semblent comme proportionnées à l'engouement qui les entoure. C'est une ferveur qui organise la «nouveauté» en «événement», témoignant que le succès de ces objets revêt aussi pour «la vie psychique» une dynamique propre au sujet. 
Pour ne pas nous laisser fasciner par cette dimension de la nouveauté, nous souhaiterions tout d'abord rendre compte de ce qui, pour la psychanalyse, fonde la logique du rapport du sujet à ses objets, ainsi qu'à leur achat. Nous partons donc de la question suivante : pourquoi l'être parlant est-il enclin à acheter des objets, et à répéter ses achats au-delà de tout besoin? Il faut pour y répondre revenir à la dialectique du désir et de la demande, telle que la psychanalyse l'enseigne.

\section{Le désir d'Autre chose}

Il y a d'abord un fait, constaté depuis toujours par les psychanalystes: la dépendance du névrosé à la demande à l'Autre, ainsi qu'à la demande de l'Autre. Le névrosé ne cesse de demander à l'Autre... ce qui lui manque, supposant et exigeant en son sein, l'objet qui lui manque. Pour autant, nous savons aussi qu'aucune des réponses de l'Autre ne suffira à le satisfaire totalement. Dès lors, entre ce qu'il demandait et ce qu'il recevra, il y aura toujours un écart, d'où surgira un manque. De ce manque, naît le désir. Telle est la raison pour laquelle en français, nous disons qu'une demande non satisfaite «laisse à désirer». Ainsi, la demande engendre le désir, lequel désir relancera ensuite la demande. Toutefois, à cela s'ajoute un paradoxe : le sujet désire aussi que sa demande ne soit pas satisfaite. Il va de soit en effet que si sa demande était totalement satisfaite, alors ce sujet ne pourrait plus continuer à désirer. Et c'est alors le manque lui-même qui viendrait à manquer, d'où surgirait l'affect d'angoisse. Ainsi, non seulement le sujet désire, mais il désire avant tout pouvoir continuer à désirer. Et c'est pourquoi le sujet ne sera jamais comblé par ce que l'Autre lui offre, même quand celui-ci satisfait sa demande. Le sujet désirera toujours Autre chose. D'où le paradoxe (Bernard, 2015, p. 39-45). Le névrosé désirera toujours Autre chose, que ce qu'il demande nommément. Autrement dit l'être parlant est un sujet toujours divisé entre ce qu'il demande et ce qu'il désire.

Le symptôme, démontre la psychanalyse, sera une façon possible d'éprouver cette division. De là, le sujet pourra alors désirer s'engager dans une psychanalyse pour déchiffrer son désir inconscient et mieux s'orienter à partir de lui. Tel est le courage du névrosé, qui dans ses paradoxes, essaye de mieux s'y retrouver. Une autre voie sera de s'en remettre à l'Autre, et de lui demander conseil pour qu'il lui suggère, voire qu'il lui ordonne quoi désirer - ce d'ailleurs sur quoi se fondent la plupart desdites «psychothérapie» 


\section{ARTIGOS}

visant «le bien du sujet», précisément celui que son symptôme ne demande pas, et auquel il objecte (Maleval, 2012; Sauret, 2008). En ce cas en effet, le sujet demandera... qu'on lui demande quelque chose. Il feint ainsi de prendre la demande de l'Autre pour l'objet de son désir, et s'aliène dans cette demande. Pensons ici à ses petits embarras au restaurant, quand le menu entre les mains, pressé de dire ce qu'il veut, il se tournera alors vers l'Autre pour lui demander quelque suggestion, c'est à dire l'offre, si ce n'est l'ordre, du jour.

Il apparaît donc une aliénation volontaire du sujet névrosé à la demande de l'Autre, à laquelle il se laisse aller pour tromper son désir. Plutôt qu'emprunter la voie de son désir, c'est-à-dire de son manque, de ce qui «laisse à désirer» et le séparerait de l'Autre, le névrosé opte volontiers pour une aliénation rassurante, ce qui pourrait être alors entendu comme une «laisse à désirer». Le maître indique alors au sujet où et comment se diriger, quitte à lui faire plusieurs propositions contradictoires, lui laissant l'illusion inhibante d'un «embarras du choix». Nous y reconnaissons un premier croisement entre la logique de la névrose et la logique capitaliste: le biennommé jeu de l'offre et de la demande. Le capitalisme aura su industrialiser la dialectique du désir et de la demande, qui n'était donc au départ qu'une invention de névrosé.

\section{L'apologue du vendeur}

Pour le montrer, Lacan (1966-1967) va alors commenter dans un petit apologue ce qu'il nomme «l'art du vendeur» (p. 204 et sq.), et tâcher d'isoler ses principes structuraux. Nous verrons cette fois non seulement comment le sujet demande à l'Autre l'objet qui lui manque et qu'il devrait désirer, mais comment il est prêt pour cela à payer, au-delà de tout besoin.

Premièrement, énonce Lacan, l'art du bon vendeur sera de «faire désirer à quelqu'un un objet dont il n'a aucun besoin, pour le pousser à le demander» (p. 210). Nous pourrions croire ici à un paradoxe: comment faire désirer à quelqu'un quelque chose dont il n'a nul besoin? Pour la psychanalyse, il y a là au contraire une cohérence. Le désir, avons-nous rappelé, est toujours désir d'Autre chose et donc justement ce qui se distingue du besoin. Et c'est pourquoi rien de tel qu'une connerie, que le superflu, que quelque chose dont on n'a nul besoin, pour faire miroiter l'objet du désir. Après quoi il s'agira en effet de pousser le sujet à demander cet objet à l'Autre, c'est à dire de l'inviter à substituer à la logique de son désir celle de la demande, selon le principe de la névrose. 
A cela, Lacan ajoute alors un second principe de l'art du vendeur. Pour convaincre l'acheteur, le vendeur devra aussi persuader le sujet que, s'il ne fait pas l'acquisition de cet objet, alors celui-ci lui manquera terriblement. L'objet derrière la vitrine aura donc aussi pour fonction d'incarner l'objet qui manquait au sujet, fut-ce à son insu. A cet égard, les écrans issus du «progrès technologique» - qui est également un argument de vente à lui seul - apparaissent comme de nouvelles vitrines, le net se faisant volontiers supermarché planétaire. ${ }^{1}$ Sony aura donné un exemple de cette logique dans l'un de ses slogans restés célèbres: «Vous l'avez rêvé? Sony l'a fait». L'industriel-vendeur se présente ainsi non seulement comme l'Autre qui saurait mieux que le sujet quel est l'objet inconscient de son désir, mais également comme celui qui saura fabriquer cet objet. Du fantasme de l'objet, nous passons ainsi directement à sa production.

À cette petite clinique de la vente et de l'achat, Lacan ajoute un troisième principe. La psychanalyse aura en effet démontré comment le désir d'un sujet est toujours connecté au désir de l'Autre. Le désir est le désir de l'Autre, dira ainsi Lacan. Le de pourra ici désigner aussi bien l'Autre en tant qu'objet désiré, que le désir de l'Autre en tant que tel, le sujet se questionnant sur ce

714 qu'il vaut pout ce désir, s'il est suffisamment aimé ou pas. Dès lors, pour faire miroiter à un sujet l'objet du désir, il faudra aussi le connecter au désir de l'Autre. Et c'est pourquoi le bon vendeur saura aussi convaincre son client que s'il n'achète pas l'objet, un autre le (lui) prendra. Cet autre aura alors ce qui lui manque, et ce faisant, aura supériorité sur lui.

\section{L'acheter, lâcheté}

De ce qui précède, nous parvenons donc à une première conclusion: l'objet ne prend sa valeur, qu'en lien avec le désir de l'Autre. "C'est par le désir de l'Autre que tout objet est présent quand il s'agit... de l'acheter» (p. 210). Dès lors, une autre question se pose: qu'achète-t-on... vraiment?

${ }^{1}$ Le succès de la plateforme de vente en ligne Amazon, dont le PDG Jeff Bezos est en 2018 l'homme le plus riche du monde, en est la parfaite illustration. Le fait qu'il ait alors dépassé Bill Gates dans ce classement semble comme symbolique: une fois l'écran et la vitrine vendue, il faut alors trouver les objets à proposer derrière, Amazon succédant ainsi comme logiquement à Microsoft et ses «Windows». 


\section{ARTIGOS}

A cette question, répond la suite du commentaire de Lacan: «L'acheter, l'acheter... lâcheté. Tiens, tiens!...» (p. 210). Lacan joue ici sur l'expression «l'acheter» en français, qui équivoque avec la lâcheté, au sens de la lâcheté morale. Il faut ici préciser que la lâcheté n'est pas à entendre comme un jugement moralisant porté sur la conduite d'achat. Il ne s'agit pas ici de juger l'acte de consommer, encore moins d'en tirer une leçon de bonne conduite qui vaudrait pour toutes et pour tous. Ici comme ailleurs dans son enseignement, Lacan entend la lâcheté au sens de l'éthique psychanalytique, où elle désigne une façon pour le sujet de ne rien vouloir savoir de son désir, ni de sa jouissance, et de se laisser égarer pour cela dans un lien d'aliénation à la demande de l'Autre. Telle est la logique même de la névrose, avons-nous indiqué plus haut, par où le sujet tentera de tromper son désir.

Ainsi, dans cet acte d'achat, où pouvons-nous situer la lâcheté? Dans ce que Lacan nomme une «malversation» (p. 210), laquelle éclaire pour lui l'un des fondements du malaise dans la civilisation, en lien avec le discours capitaliste. Il se produit en effet une malversation dans la façon par laquelle le sujet tente de substituer à son désir, ce que l'Autre lui demande d'acheter. A ce désir qui faisait sa singularité, le sujet s'égarera en s'aliénant à ce que l'Autre lui suggère d'acheter, c'est à dire en se conformant sagement à ce que l'Autre marchand lui demande d'avoir. Lacan en souligne alors la conséquence. À suivre cette voix trompeuse de l'achat, le sujet sera toujours poussé plus loin à devoir se racheter. Il précise, usant pour cela du tutoiement propre à l'éthique pour réveiller le sujet: «De te racheter de ta lâcheté» (p. 210). Au terme de cet apologue de l'art du vendeur, voici donc que se dévoile la thèse de Lacan. A la dette symbolique qui fondait le désir à l'appui d'un manque irrémédiable, s'oppose la dette moderne que le sujet pourrait racheter. Il s'agirait ici de s'affranchir de la dette symbolique en la rabattant sur la dette financière, cette dette que l'on pourrait racheter. Tentative impossible, démontre Lacan, du fait que la dette symbolique est due au fait que l'on parle, tout simplement. Dès lors, la tentative sera toujours réitérée. Acheter est tenter de racheter sa lâcheté, encore et encore.

Le sujet, s'aliénant aux promesses de bonheur du discours capitaliste, s'égarera ainsi toujours plus au regard de son désir singulier, et ne pourra alors qu'en éprouver les effets de retours dans ses symptômes. L'affect de culpabilité était pour Lacan l'un des affects signes de cet égarement, auquel il ajoutera la honte, la morosité, l'ennui, l'angoisse. Autant d'affects que le sujet pourra fuir en allant toujours plus loin dans la malversation première. Voilà donc aussi l'un des principes de la réussite du capitalisme: acheter pour 
se déculpabiliser, se désangoisser, se divertir, voire «faire diversion» face à sa propre question, son propre insupportable, selon l'expression de Freud (1929/1971, p. 17-18) dans son Malaise.

\section{Acheter... n'importe quoi}

Lacan y reviendra plus tard dans un autre texte intitulé «Radiophonie». Quand on s'ennuie, remarque-t-il, «on (...) achète n'importe quoi» (Lacan, 1970/2001, p. 414). Nous voudrions à présent insister sur cette articulation entre la cause de désir, et ce «n'importe quoi», pour isoler une autre thèse de Lacan concernant cette exploitation capitaliste du désir. Il y a en effet des raisons précises pour lesquelles il souligne ici que l'on achète... n'importe quoi. Ainsi que nous l'évoquions plus haut, «n'importe quoi» dit que cet objet sera distinct d'un objet dont on aurait vraiment besoin. Toutefois, l'expression dit aussi que peu importe l'objet en question, quand l'essentiel est ailleurs: pouvoir tromper le désir dont l'ennui était le signe.

Voilà qui rejoint une autre remarque de Lacan, visant également la façon dont un sujet, pour s'orienter, peut s'en remettre aveuglément à l'Autre: «Toute la politique repose sur ceci, que tout le monde est trop content d'avoir quelqu'un qui dit En avant marche - vers n'importe où, d'ailleurs» (Lacan, 1985, p. 5-23). Vers n'importe où, du moment que le sujet puisse être ainsi soulagé de la responsabilité et de la question de son désir. Ainsi Aristide Boucicaut, fondateur du «Bon marché» de Paris à la fin du xixème siècle, et pionnier du «grand magasin» avait compris qu'en disposant «n'importe où» ses marchandises, c'est-à-dire sans rayonnements bien délimités, réfléchis, logiques, les gens finiraient bien par acheter «n'importe quoi», et donc, toujours plus. La recette n'a pas démenti, et l'objet, le manque qu'il recouvre, commande l'achat. Le névrosé est ainsi, de structure, «sans force contre la réclame» (Zola, 1883/2009, p. 301) selon l'expression d'Octave Mouret, personnage par lequel Zola avait croqué Boucicaut dans $\mathrm{Au}$ bonheur des dames.

Nous voici donc avec ces deux expressions, disant chacune cet égarement moderne du sujet: «N'importe quoi», aussi bien que «Vers n'importe où». Les deux expressions méritent en effet d'être associées l'une à l'autre dans la mesure où dans le discours capitaliste, poursuit-il, l'objet de consommation est bien ce qui viendra finalement commander au sujet. Tel est en effet pour Lacan le secret du pouvoir capitaliste. Dire que ce discours 


\section{ARTIGOS}

est parvenu à exploiter le désir, est dire aussi qu'il est parvenu à mettre aux commandes l'objet de consommation lui-même. La thèse est explicite, dans son texte «Radiophonie». L'exploitation qui définit le discours capitaliste est déterminée non pas par des maîtres, mais par les "produits» eux-mêmes. C'est donc à ces produits que les sujets «pourraient demander compte de l'exploitation qu'ils subissent» (Lacan, 1970/2001, p. 435).

Il est intéressant de constater qu'aujourd'hui, les objets dits «intelligents» ou «connectés» sont susceptibles de pouvoir donner des indications de cette exploitation. Le cyberespace a très tôt illustré ce «n'importe quoi», voire ce «n'importe où», caractéristique du cheminement de l'être parlant, comme de son égarement à l'occasion. «Si c'est gratuit, c'est que tu es le produit» dit le fameux dicton du web. Effectivement, c'est peut-être sur ce point de la suggestion, du conseil, voire de la «recommandation» que peut particulièrement se saisir le croisement du capitalisme et du numérique. Les algorithmes commerciaux, utilisés par les moteurs de recherches et les plateformes commerciales en ligne, l'illustrent. Cette transformation de l'internaute en «produit» par l'extraction de diverses informations fait parfois retour via le «scandale» ${ }^{2}$ (Pixel, 2018), dont la force indique finalement le degré d'aveuglement qu'avait provoqué cette aliénation, la «force de l'écran» à nous faire «fermer les yeux». C'est peut-être là le secret de toute publicité. «Nous créons l'impensable pour que vous réalisiez l'impossible» clamait un spot pour un casque de réalité virtuel de la marque Samsung. ${ }^{3}$ Effectivement, si le publiciste "vend l'impensable», le consommateur lui ne peut que s'affronter à l'impossible, sauf à faire l'autruche, comme le suggérait finement l'animal choisi pour illustrer le message. Sa traduction anglaise laisse encore moins de doute, puisqu'ordonner au spectateur: «Do what you can't», c'est-à-dire littéralement, «Fais ce que tu ne peux pas faire», ne peut relever que de la politique de cet animal.

${ }^{2}$ Les exemples sont multiples, un des derniers en date, et parmi les plus marquant est sans doute celui des extractions de données via la plateforme Facebook pour les campagnes présidentielles américaines de 2016.

${ }^{3} \mathrm{Cf}$. Le spot publicitaire de la marque Samsung «Do What You Can't» [en ligne : https://www.youtube.com/watch?v=T5FXYPBmcoY] (consultée le 12.02.2019). 


\section{Addicts}

La publicité, dans sa «vérité-menteuse» («Do what you can't!») prend la forme d'une réalité virtuellement repoussée à l'infini, celle de l'objet satisfaisant tous les désirs. C'est en définitive le crédo même du libéralisme, dont le pionnier qu'était Adams Smith, dans la théorisation de la légendaire «main invisible» indiquait qu'à satisfaire l'Autre on se trouverait également satisfait: «Donnez-moi ce dont j'ai besoin et vous aurez de moi ce que vous avez besoin vous-mêmes» ${ }^{4}$ (Lesourd, 2011, p. 183). Mais les besoins du Moi ne sont pas à confondre avec le désir du sujet, toujours déjà perdu et recherché chez l'Autre, faute de quoi l'échange se trouverait arrêté dès la satisfaction rencontrée. Le libéralisme «magique» d'Adams Smith joue à méconnaître la «konstant kraft» (poussée constante) de la pulsion freudienne (Freud, 1915/1986). En revanche, lesdits «annonceurs», eux, ne l'ignoraient pas.

En effet, la publicité organise aujourd'hui le financement de la majorité des plateformes qui proposent «gratuitement» leurs services aux internautes. Cette pratique interroge économistes, sociologues, ingénieurs depuis ses débuts. Certains pensaient même que tout ce qui serait numérique serait gratuit dans un avenir toujours... à venir. Le slogan «si c'est gratuit, c'est que tu es le produit» (Anderson \& Le Séac'h, 2009), éclaire autrement la dynamique. Ce slogan pourrait, au-delà de l'économie efficace de sa formule, trouver d'autres vérifications. Notamment dans l'exploitation algorithmique de nos traces, véritable excavation d'un nouveau minerai et dont il s'agit de définir le prix. Mais s'il n'a pas encore de prix, le principe répond à une logique: après avoir réduit notre pratique des objets numériques à des «profils», c'est-à-dire à des indicateurs calculables et comparables, les algorithmes (de suggestion d'achats... ou de vote!) nous présentent $\mathrm{du}$ contenu «susceptible d'intéresser», ainsi que le veut l'euphémisme de Facebook. Le projet de ces algorithmes marchands est bien de trouver l'objet correspondant à la «cible» (puisqu'on parle alors de «publicité ciblée», la cible n'étant évidemment pas la publicité elle-même, mais bien le consommateur — et ici l'internaute). En d'autres termes, par les traces numériques que laissent sur le net nos chemins empruntés, les publicitaires

${ }^{4}$ Selon la célèbre phrase attribuée à Adam Smith dans son ouvrage De la richesse des nations. 


\section{ARTIGOS}

et commerciaux tentent de deviner l'objet de notre désir, celui qui nous intéresserait et que nous serions susceptible d'acheter, donc (Thomas, 2016). G. Berry, premier occupant de la chair d'informatique du Collège de France indique entre les lignes de son ouvrage la déception du scientifique qu'il est dans l'application de ses inventions à des fins mercantiles. Il souligne aussi comment aujourd'hui, l'appréhension de nos désirs par les machines paraît grossière. Il relève ainsi que les objets «suggérés» par les algorithmes sont souvent, en réalité, déjà achetés depuis longtemps, ou qu'ils ne sont que le résultat de simple collage à partir des «métadonnées» issues de recherches dissociées, ou d'informations erronées, etc. (Berry, 2017, p. 137). La raison numérique propose une empreinte, un profil en creux, de l'internaute. Mais sa «patte» de sujet, elle, file toujours entre les doigts du calcul de l'Autre capitaliste, ouvrant alors à la répétition.

Si nul ne peut remettre en cause le succès de ces pratiques auprès des consommateurs, force est de constater que ces nouvelles techniques et objets amènent à de nouveaux symptômes, de nouveaux ratages. Beaucoup de psychanalystes considèrent aujourd'hui que le sujet moderne consommerait une jouissance sans limite, du fait des gadgets produits par la science. La modernité serait ainsi caractérisée par cette jouissance que rien ne viendrait restreindre. La thèse de Lacan est pourtant toute autre. Si jouissance illimitée il y a, celle-ci n'est pas celle que les gadgets surconsommés offriraient. Ce qui au contraire aliènera le sujet dans cette surconsommation sera ce que Lacan nomme «la soif du manque à jouir» (Lacan,1970/2001, p. 435). Qu'est-ce à dire? Premièrement, que contrairement à ce qu'il promet, le discours capitaliste s'entretient de ce manque à jouir. Le sujet fera en effet l'expérience, dans la consommation, d'un manque, dans la mesure où l'objet qu'il s'achète et qu'il consomme n'équivaudra jamais à celui qui causait son désir. Toutefois une autre jouissance, dans cette expérience, pourra se consommer: la jouissance du manque. Si donc il y a bien ici une jouissance qui se satisfait de façon illimitée, il s'agit en fait de cette jouissance du manque à jouir, de cette jouissance de la perte, d'une limite sans cesse répétée. Ça n'est pas (encore) çà, raison pour laquelle on recommence, encore et encore. Et c'est pourquoi l〉objet de consommation est fondamentalement un objet en série.

Se payer l'iphone 10, est se payer le manque de l'iphone 11. Le philosophe Günther Anders l'avait depuis longtemps démontré (Anders, 1956/2002): le consommateur, qui croit posséder, n'est propriétaire de rien. Celui qui se paye le dernier cri, se paye le manque de l'objet suivant. 
La réussite du capitalisme fut aussi d'avoir su donner à cette jouissance du manque le masque d'un plus, d'un toujours plus. Tel est le principe de la série: Encore une, faisant du consommateur type, un serial-consommateur. Il s'éclaire alors la raison pour laquelle l'addiction est devenue aujourd'hui l'un des symptômes majeurs dans les sociétés capitalistes. La théorisation lacanienne nous permet toutefois de préciser que ce à quoi le sujet est addict n'est pas la jouissance de l'objet en tant que telle, mais la jouissance répétée du manque de bon objet, la jouissance du «Un peu plus et ça y était». Et c'est pourquoi en effet, la jouissance qu'entretient le discours capitaliste n'est pas à situer dans tel ou tel gadget moderne, mais dans ce toujours plus. Autrement dit dans ce plus, toujours. D'où le terme ici choisi par Lacan pour désigner cette jouissance de la série: «insatiable» (Lacan,1970/2001, p. 435), lequel terme désigne en français un désir qui ne peut jamais être comblé. La réussite du discours capitaliste, contrairement à ce qu'il nous fait miroiter, n'est donc pas de combler le désir, mais d'exploiter le fait qu'il ne peut être comblé. Le principe même de l'économie capitaliste est d'exploiter la jouissance du ratage, en série.

\section{Conclusion}

Nous avons ainsi tâché de démontrer dans ce qui précède les raisons pour lesquelles, à suivre l'enseignement du psychanalyste Jacques Lacan, le sujet moderne, ledit consommateur, ne peut être décrit comme un sujet comblé par ses objets, atteignant à une jouissance sans limite. Il s'agit là plutôt de la fausse promesse du discours capitaliste, qui dans ses slogans publicitaires ne cesse en effet de vendre du sans limite. Sa réussite aura été cependant d'avoir su exploiter plus secrètement la structure du désir, qui elle repose au contraire sur la répétition du manque, et la jouissance que cette répétition procure. Plus fondamentalement, sa réussite aura été d'avoir su exploiter la logique de la névrose, la substitution de l'objet de la demande à la cause du désir. Il s'en déduit pour la psychanalyse l'importance de reconnaître, dans cette exploitation, la responsabilité du sujet lui-même. Il n'y a d'exploitation du désir, qu'à la condition d'un sujet qui, souvent inconsciemment, se laisse exploiter (Lacan,1970/2001, p. 415) par les produits qu'on lui propose. 


\section{ARTIGOS}

\section{References}

Anders, G. (2002). L’obsolescence de l’homme. Paris, França: Ivrea. (Travail original publié dans 1956).

Anderson, C., \& Le Séac'h, M. (2009). Free! entrez dans l'économie du gratuit. Paris, França: Pearson-Village mondial.

Berry, G. (2017). L'hyperpuissance de l'informatique: algorithmes, données, machines, réseaux. Paris, França: Odile Jacob.

Breton, P. (1990). Une histoire de l'informatique. Paris, França: Seuil.

Bernard, D. (2015). Paradoxes du désir et de la demande. Revue de l'École de Psychanalyse des Forums du champ lacanien, 16, 39-45.

Thomas, J. (2016). Les algorithmes du désir: que fait la raison numérique de nos traces?. La Cause du désir, 94.

Freud, S. (1971). Malaise dans la civilisation. Paris, França: PUF. (Travail original publié dans 1929).

Freud, S. (1986). Pulsion et destins des pulsions. In Métapsychologie. Paris, França: Gallimard. (Travail original publié dans 1915).

Lacan, J. (1966-1967). Séminaire la logique du fantasme. Leçon du 21/06/1967, inédit. [repéré à: http://staferla.free.fr/S14/S14\%20LOGIQUE.pdf, consultée le 12.02.2019].

Lacan, J. (1978). «Intervention dans une réunion organisée par la Scuola freudiana», à Milan, le 4 février 1973. Lacan in Italia 1953-1978. In Italie Lacan (pp. 78-97). Milan, Italia: La Salamandra.

Lacan, J. (1985). «Conférence à Genève sur le symptôme», prononcée le 4 Octobre 75. Le Bloc-notes de la psychanalyse, 5, 5-23.

Lacan, J. (1991). Le séminaire. Livre XVII. L'envers de la psychanalyse. Paris, França: Seuil. (Travail original publié dans 1969-70).

Lacan, J. (2001). Radiophonie. In Autres écrits. Paris, França: Seuil. (Travail original publié dans 1970).

Maleval, J.-C. (2012). Étonnantes mystifications de la psychothérapie autoritaire, Paris, França: Navarin Le Champ freudien.

Pixel (la rédaction) (2018). «Cambridge Analytica: 87 millions de comptes concernés». Le Monde, le 4 avril 2018. [repéré à: https:/www.lemonde.fr/pixels/ article/2018/04/04/cambridge-analytica-87-millions-de-comptes-facebookconcernes_5280752_4408996.html, consultée le 07/11/2018].

Lesourd, S. (2011). La fermeture des espaces du je: Le discours du libéralisme. Revue internationale de psychosociologie, XVII(43), 183. 
Sauret, M. J. (2008). L'effet révolutionnaire du symptôme. Ramonville Saint-Agne, França: Erès.

Zola, É. (2009). Au bonheur des dames. Paris, França: Fasquelle. (Travail original publié dans 1883).

\section{Résumés}

(Desejo, compra e consumo: uma abordagem lacaniana)

O presente estudo tem como objetivo questionar a função que podem ocupar, para o sujeito falante, as práticas de compra e consumo, ainda pouco estudadas como tal no campo da psicanálise e da psicopatologia. A partir do ensino de Jacques Lacan, tentaremos esclarecer a lógica dessas práticas, precisando como elas podem constituir, para um sujeito, uma resposta falsa diante do embaraço de seu desejo. Em seguida, questionaremos igualmente como essas práticas se atualizam no espaço digital do mundo contemporâneo e como, ao fazê-lo, elas participam do atual mal- estar na civilização.

Palavras-chave: Comprar, consumir, desejo, laço social

(Desire, purchasing, consumption: A Lacanian approach)

This paper questions the function that purchasing and consumption practices can have for the speaking being. So far, these practices have been little studied as such in the field of psychoanalysis and psychopathology. Based on Jacques Lacan's teaching, we will try to clarify the logic behind those practices, by identifying in what way they can provide a subject with a false answer to the embarrassment of his desire. Then, we will also question how those practices are updated in the digital space of the contemporary world, and how they contribute to the current discontent in civilization.

Key words: Purchasing, consumption, desire, social bond

(Deseo, compra y consumer. un nfoque lacaniano)

Se tratará de interrogar aqui la función que pueden ocupar, para el ser que habla, las prácticas de compra y de consumo, aún poco estudiadas en el campo del psicoanálisis y de la psicopatología. Desde de la enseñanza de Jacques Lacan, trataremos de aclarar la lógica de dichas prácticas, estableciendo cómo pueden constituir, para un sujeto, una falsa respuesta frente a lo confuso de su deseo. Desde ahí, nos interrogaremos también sobre cómo estas prácticas se actualizan en el espacio numérico del mundo contemporáneo y cómo, al hacerlo, contribuyen con el malestar actual de la civilización.

Palabras clave: Comprar, consumir, desear, lazo social 


\section{ARTIGOS}

(Begehren, kaufen, konsumieren:ein Lakanischer Ansatz)

Ziel dieses Artikels ist es, jene Funktion zu hinterfragen, die Kauf und Konsum für das sprechende Wesen darstellen, Handlungen, die als solche in der Psychoanalyse und Psychopathologie bisher wenig erforscht wurden. Auf der Grundlage der Lehre Jacques Lacans soll versucht werden, die Logik dieser Handlungen zu beschreiben und dabei herauszuarbeiten, inwiefern sie für das Subjekt eine falsche Antwort auf die Verlegenheit angesichts seines Begehrens darstellen könnte. Davon ausgehend wird dann erörtert, wie sich diese Handlungen im digitalen Raum unserer Gegenwart aktualisieren und wie sie eben dadurch zum heutigen Unbehagen in der Kultur beitragen.

Schlüsselwörter: Kaufen, konsumieren, begehren, soziale Bindung

Citação/Citation: Bernard, D., \& Dumoulin, Q. (2019, dez.). Désirer, acheter, consommer: approche lacanienne. Revista Latinoamericana de Psicopatologia Fundamental, 22(4), 710724. http://dx.doi.org/10.1590/1415-4714.2019v22n4p710.4.

Editora/Editor: Profa. Dra. Sonia Leite

Submetido/Submitted: 6.3.2019/3.6.2019 Aceito/Acepted: 4.10.2019/10.4.2019

Copyright: (C) 2009 Associação Universitária de Pesquisa em Psicopatologia Fundamental/ University Association for Research in Fundamental Psychopathology. Este é um artigo de livre acesso, que permite uso irrestrito, distribuição e reprodução em qualquer meio, desde que o autor e a fonte sejam citados / This is an open-access article, which permits unrestricted use, distribution, and reproduction in any medium, provided the original authors and sources are credited.

Financiamento/Funding: Este trabalho não recebeu apoio / This work received no funding. Conflito de interesses/Conflict of interest: Os autores declaram que não há conflito de interesses. / The authors declare that there is no conflict of interest. 
DAVID BERNARD

Maître de conférences HDR (Habilitation à diriger des recherches) en psychopathologie à l'Université Rennes 2 (France), EA4050.

dabernard2@yahoo.fr

https://orcid.org.0000-0003-0502-1110

Quentin Dumoulin

Psychologue clinicien; doctorant contractuel, chargé de cours à l'Université Rennes 2 (France) EA4050.

Département de psychologie / UFR Sciences Humains

Université Rennes 2

Place du recteur Henri Le Moal

35043 Rennes, France

quentindumoulin@yahoo.fr

https://orcid.org.0000-0002-6221-1482

This is an open-access article, which permits unrestricted use, distribution, the original authors and sources are credited. 